\title{
Article \\ Numerical Investigations on the Shape Optimization of Stainless-Steel Ring Joint with Machine Learning
}

\author{
Minsoo Kim ${ }^{1}$, Sarang $\mathrm{Yi}^{2}$ and Seokmoo Hong ${ }^{1,2, *(D)}$ \\ 1 Department of Mechanical and Automotive Engineering, Kongju National University, Cheonan 31080, Korea; \\ mkim@kongju.ac.kr \\ 2 Department of Future Convergence Engineering, Kongju National University, Cheonan 31080, Korea; \\ olluv@smail.kongju.ac.kr \\ * Correspondence: smhong@kongju.ac.kr; Tel.: +82-(0)41-521-9268
}

Citation: Kim, M.; Yi, S.; Hong, S.

Numerical Investigations on the Shape Optimization of Stainless-Steel Ring Joint with Machine Learning. Appl. Sci. 2021, 11, 223.

https://doi.org/10.3390/ app11010223

Received: 2 December 2020

Accepted: 24 December 2020

Published: 28 December 2020

Publisher's Note: MDPI stays neutral with regard to jurisdictional claims in published maps and institutional affiliations.

Copyright: (c) 2020 by the authors. Licensee MDPI, Basel, Switzerland. This article is an open access article distributed under the terms and conditions of the Creative Commons Attribution (CC BY) license (https: / / creativecommons.org/ licenses/by/4.0/).
Abstract: Since pipes used for water pipes are thin and difficult to fasten using welding or screws, they are fastened by a crimping joint method using a metal ring and a rubber ring. In the conventional crimping joint method, the metal ring and the rubber ring are arranged side by side. However, if water leaks from the rubber ring, there is a problem that the adjacent metal ring is rapidly corroded. In this study, to delay and minimize the corrosion of connected water pipes, we propose a spaced crimping joint method in which metal rings and rubber rings are separated at appropriate intervals. This not only improves the contact performance between the connected water pipes but also minimizes the load applied to the crimping jig during crimping to prevent damage to the jig. For this, finite element analyses were performed for the crimp tool and process analysis, and the design parameters were set as the curling length at the top of the joint, the distance between the metal rings and rubber rings, and the crimp jig radius. Through FEA of 100 cases, data to be trained in machine learning were acquired. After that, training data were trained on a machine learning model and compared with a regression model to verify the model's performance. If the number of training data is small, the two methods are similar. However, the greater the number of training data, the higher the accuracy predicted by the machine learning model. Finally, the spaced crimping joint to which the derived optimal shape was applied was manufactured, and the maximum pressure and pressure distribution applied during compression were obtained using a pressure film. This is almost similar to the value obtained by finite element analysis under the same conditions, and through this, the validity of the approach proposed in this study was verified.

Keywords: stainless-steel ring joint; support vector machine; finite element analysis; statistical regression; optimization

\section{Introduction}

Functions such as light-weighting and corrosion resistance are being strengthened, whereas construction convenience must be considered when connecting pipes such as water or gas pipes. In particular, it is difficult to apply welding and fastening methods using screws because pipes used as water pipes are thin. Therefore, a crimping joint method using a pressure jig is applied when connecting water pipes. A crimping joint with dual ring points, which is a type of crimping joint, is shown in Figure 1. The joints on the ends of the two pipes to be connected are compressed when a metal ring for preventing vibration and deflection and a watertight rubber ring are inserted between the joints and the connecting pipe. When the material of the metal ring inside the joint is stainless steel, it is known as the stainless ring (SR) joint, as shown in Figure 1a. The commercial crimping joint with dual ring points contains a metal ring and a rubber ring adjacent to it Figure 1b; hence, the two rings can be compressed using a relatively small pressing tool in a single attempt. Although such a structure affords the light-weighting of the tool or high work efficiency, the rubber ring might penetrate into the dented part of the metal ring after the compression, 
as shown in Figure 1c. Consequently, leaked water due to the lowered water-tightness accumulates in the gap between the fitted pipe and SR-joint, resulting in corrosion. In addition, a conventional SR-joint cannot respond to the recently reinforced seismic design of structures owing to problems such as the separation of rubber rings or rotation of metal rings when vibrations occur because of earthquakes. A new SR-joint shape that separates the metal and rubber rings must be developed for improvement. Yoon et al. [1] conducted structural analysis of the crimping tool in the crimping state as a preliminary preparation stage for the technology development of the SR-joint. However, there is a limitation that the analysis of various parameters affecting pipe compression is insufficient, and actual verification has not been performed. Although empirical design is being conducted by observing the pipe joint and the cut surface at the field, the behavior of the metal and rubber rings inside the pipe is not well understood when the SR-joint is compressed. Therefore, it is difficult to develop and apply a new SR-joint that separates the metal and rubber rings. Various studies have been conducted to derive an optimal design method using finite element analysis to solve problems encountered in such an empirical design. Onyegiri et al. [2] investigated the mechanical deformation between pipes during the field joining of a bent sandwich pipe. Li et al. [3] analyzed the load for a $180^{\circ}$ pipe bending using three-dimensional (3D) finite element analysis considering geometric nonlinearity. In addition, Lee et al. [4] performed a contact analysis of wedge-type pipe joints, whereas Lee et al. [5] developed plastic deformation and permanent crimping methods for pipes using pressing tools based on finite element analysis. Lee et al. [6] predicted the wear life of the $\mathrm{CrN}$ coating layer on a press tool for stamping an ultra-high-strength steel sheet through a design of experiment. Hosseinpour et al. [7] applied a design of experiment to analyze the performance of the internal fin shape of a microchannel heat sink and then optimized the shape.

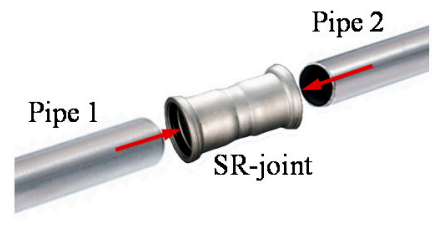

(a)

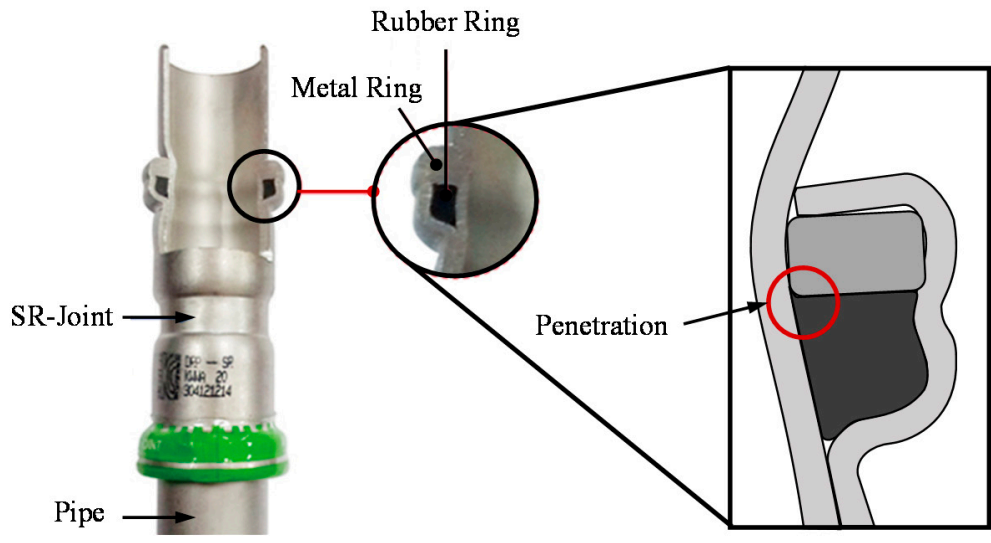

(b)

(c)

Figure 1. (a) Principle of stainless ring (SR) joint connection (b) Combined shape and cross-section of pipe and joint (c) An enlargement of the dual ring part.

Regression analysis has been performed to obtain the optimal design of a product, as in the aforementioned studies; however, studies using artificial intelligence have increased recently to further improve the accuracy of prediction models. Son et al. [8] used finite element analysis in addition to machine learning to develop a model for predicting the surface deflection of rectangular drawing products. Kwak et al. [9,10] developed a model to design an optimal initial blank shape by applying an artificial neural network in a rectangular case formed with large aspect ratio; furthermore, they developed longitudinal and transverse spring-back prediction models using an artificial neural network in the multipoint die-less forming of advanced high strength steel. Woo et al. [11] applied an artificial neural network model to obtain constitutive equation parameters of materials in high-speed forming processes to obtain material properties based on the strain rate. Park and Kang [12] predicted the curvature of the roll-forming process using flexible 
reconfigurable roll forming technology and compared the conventional regression model with the artificial neural network model. Wang et al. [13] predicted the bending force in the hot rolling process using an artificial neural network and a genetic algorithm and then applied the proposed model for online control and schedule optimization. Park et al. [14] developed a model for the numerical prediction of temperature-dependent flow stress on fiber metal laminates using an artificial neural network; they demonstrated that the model-predicted results were more accurate than conventional numerical results. Olazagoitia et al. [15] showed that artificial neural networks can be used to directly identify the parameters of a tire model for a real test without iterative fittings or initial iteration point definitions without using complex cost functions. Mei et al. [16] used the deep neural network model to accurately characterize the nonlinear relationship between the parameters of the Inconel 718 bonding process and bonding performance. Hijazi et al. [17] used artificial neural networks modeling to predict the residual stress of aluminum panels using multiple site damage, showing that it can predict with high accuracy the residual strength for all materials and constructions considered in the study.

A new SR-joint shape (SR-II) that separates a metal ring from a rubber ring from the conventional joint is proposed herein to reduce corrosion and improve water tightness. The concepts of the conventional method (SR-I) and the proposed SR-joint (SR-II) were compared, as shown in Figure 2. To improve the lifetime of the pressing tool, variables that can be changed in the actual process were selected, and various combinations of finite element analysis were conducted to minimize the load on the pressing tool during the compression. The optimal design derived via finite element solution-based regression analysis and the optimal solution predicted by machine learning techniques were compared to analyze the effectiveness of the optimal design based on the number of analyses considered. Data to be trained in machine learning were acquired from simulations for various cases and were used for training using a support vector machine (SVM). Finally, the result of the study was verified by measuring the contact pressure after the optimal design shape was fabricated.

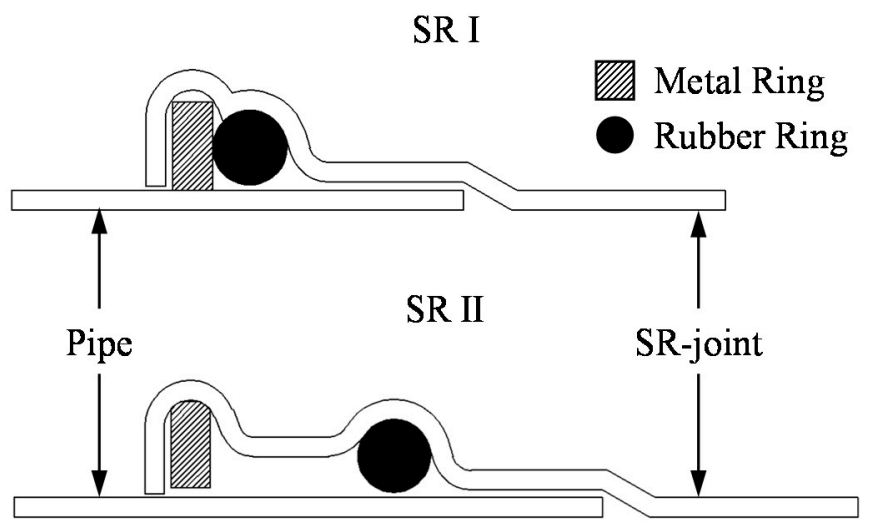

Figure 2. Schematic diagram of SR-joint.

\section{Development of an Advanced SR-JOINT (SR-II)}

\subsection{Finite Element Analysis}

\subsubsection{Design of a New SR-Joint (II) and Material Properties}

An SR-joint structure is composed of an inner pipe, an SR-joint, a metal ring, and a rubber ring, similar to the conventional SR-I and the proposed SR-II, as shown in Figure 2. SUS304 is generally used for pipes, SR-joints, and metal rings, whereas ethylene propylene diene monomer rubber (EPDM) is used for rubber rings [18]. Material tests of SUS304 and EDPM were conducted to obtain material properties to be used in finite element analysis. EDPM was tested using compressed specimens with diameters and heights of $29 \mathrm{~mm}$ and $12.7 \mathrm{~mm}$, respectively, because the rubber ring deforms under compression force. The specimen shape of SUS304 complied with the KS B 0801 standard [19], and tensile and compression tests were performed at a displacement-controlled load speed 
of $4 \mathrm{~mm} / \mathrm{min}$ using the universal testing machine SHIMADZU AG-X. The mechanical properties obtained through tensile and compression tests are shown in Table 1 . The flow stress curve of the pipe material is expressed as shown in Equation (1) using the Swift equation based on the results of tensile and compression tests [20].

$$
\bar{\sigma}=k\left(\bar{\varepsilon}+\varepsilon_{0}\right)^{n}
$$

Table 1. Mechanical properties of SUS304 and ethylene propylene diene monomer rubber (EPDM).

\begin{tabular}{ccc}
\hline Material & SUS304 (Stainless Steel) & EDPM (Rubber) \\
\hline$k[\mathrm{MPa}]$ & 1426 & 6.018 \\
$\varepsilon_{0}[-]$ & 0.029 & 0.001 \\
$n[-]$ & 0.502 & 1 \\
Young's Modulus [GPa] & 207 & 15 \\
Poisson's ratio [-] & 0.28 & 0.49 \\
Yield Stress [MPa] & 215 & 0.0058 \\
\hline
\end{tabular}

In the equation, $\bar{\sigma}$ denotes the effective stress, $k$ denotes the strength coefficient, $\bar{\varepsilon}$ denotes the effective strain, $n$ denotes the strain-hardening exponent, and $\varepsilon_{0}$ denotes the initial strain. Figure 3 shows the specimen shape and true stress-true strain curve of each material.
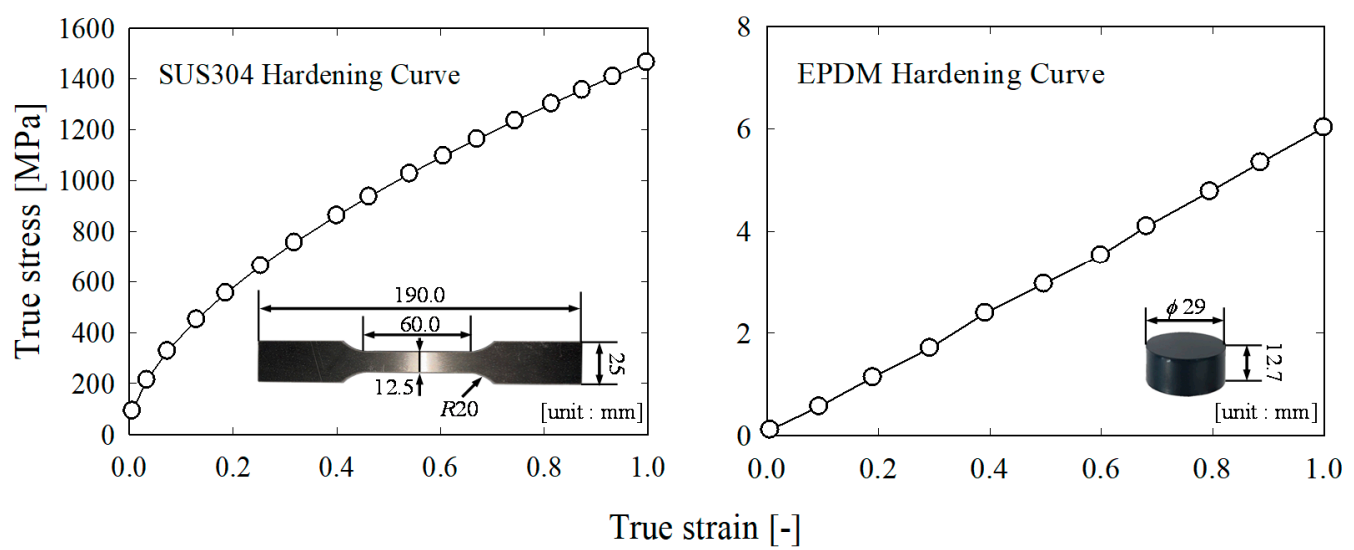

Figure 3. Specimen shapes and true stress-true strain curves of SUS304 and EPDM.

\subsubsection{Finite Element Analysis of SR-Joint (SR-II)}

A finite element analysis was performed on the conventional pipe crimping process to analyze the validity of the finite element analysis program. Analysis modeling was performed using AFDEX V20, a commercial finite element analysis program, to analyze the conventional SR-joint molding behavior. Although finite element analysis is typically performed with 3D modeling in principle, because the pipe had the same revolved section based on the central axis, a two-dimensional axisymmetric finite element model was constructed instead. The conventional SR-joint is a structure in which a metal ring and a rubber ring are adjacent to each other between the SR-joint that has completed the expansion process and the pipe that has not been processed; furthermore, they are fitted as shown in Figure 4 . An image showing only the $270^{\circ}$ view to visualize the inside of the material is shown in Figure $4 \mathrm{a}$, and the cross-sections of the pressure jig, pipe, metal ring, and rubber ring, and two fitted pipes are shown in Figure $4 \mathrm{~b}$. The fitted shape after compression and finite element analysis is shown in Figure 4c. The shape considered in this study is a stainless-steel pipe with a diameter and thickness of 114 and $2 \mathrm{~mm}$, respectively. The rubber ring has a circular cross-section and a diameter of $7 \mathrm{~mm}$, and the metal ring 
has a rectangular cross-section and $8.35 \mathrm{~mm}$ and $3 \mathrm{~mm}$ in width and length, respectively. Finite element modeling for pipes, SR-joints, metal rings, and rubber rings was made using 4-node elements of about 1020, 770, 210, and 920, respectively. Since the plastic deformation of the metal ring and rubber ring is relatively large during compression, many elements are placed. In the order mentioned above, the minimum element size for each part is $0.666 \times 1.065,0.349 \times 0.663,0.141 \times 0.141$, and $0.136 \times 0.136 \mathrm{~mm}^{2}$, respectively. Since the difference in pipe diameter before and after the actual compression process was $8.8 \mathrm{~mm}$, the pressure jig stroke was set to $4.4 \mathrm{~mm}$, which was half the measured value in consideration of the axisymmetric shape in the $x$-axis direction. The speed of the pressure jig was $-1 \mathrm{~mm} / \mathrm{s}$ in the $x$-axis direction. Considering the analysis time and convergence, the pressure jig was assumed to be a rigid body.

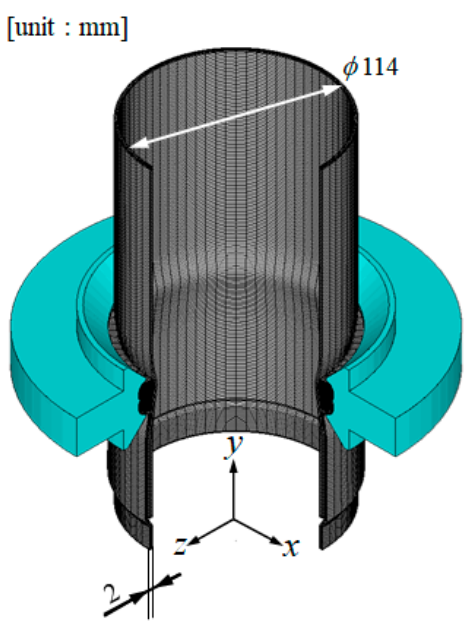

(a)

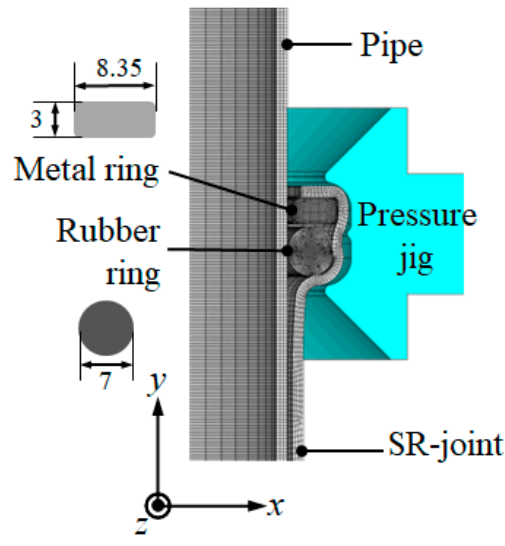

(b)

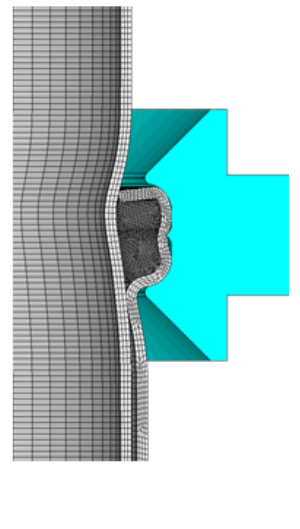

(c)

Figure 4. Geometry and FE modeling of a conventional stainless ring (SR)-joint (SR-I). (a) overall mesh design (b) detailed dimensions for each part (c) deformed shape of each part after compression.

The finite element analysis results of the conventional SR-joint are as shown in Figure 5, and the maximum molding load was $340.8 \mathrm{kN}$. The maximum pressure applied to the pressure jig was calculated using Equation (2) based on the load applied per unit area, where $P$ denotes the pressure, $F$ denotes the load $[\mathrm{N}]$, and $A$ denotes the cross-sectional area $\left[\mathrm{mm}^{2}\right]$.

$$
P=F / A
$$
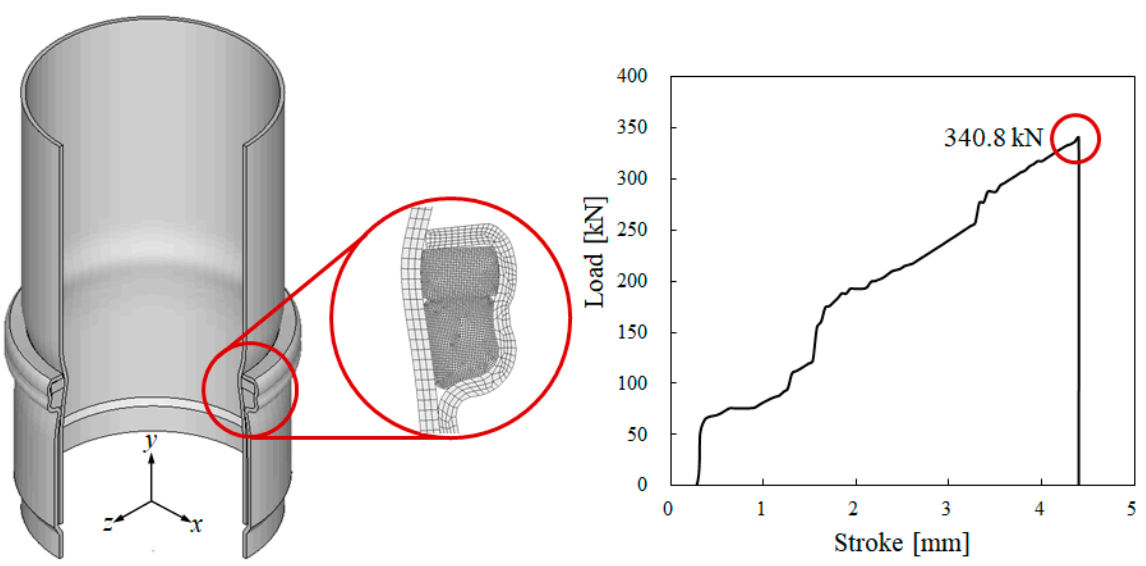

Figure 5. Numerical load-stroke curves of the conventional SR-joint (SR-I). 
After the finite element analysis, the contact area between the pressure jig and SR-joint was measured using a 3D-computer aided design (CAD) program, and the calculated contact area was $154.54 \mathrm{~cm}^{2}$. If the maximum load value of $340.8 \mathrm{kN}$ and a contact area of $154.54 \mathrm{~cm}^{2}$ derived through finite element analysis are substituted into Equation (2), then the pressure obtained will be $22.05 \mathrm{MPa}$ at $2.205 \mathrm{kN} / \mathrm{cm}^{2}$. This shows that the SR-joint can be molded within $30 \mathrm{MPa}$, which is the maximum hydraulic pressure applied during compression molding in the field.

\subsection{Optimal SR-Joint Shape Design}

\subsubsection{Optimal Shape Design Variables}

The new SR-joint shape (SR-II) was designed to prevent corrosion by placing a gap between the rubber and metal rings. The initial shape of SR-II designed using field experience was compared with the conventional SR-I shape through finite element analysis. The results of the SR-II initial shape analysis shown in Figure 6 indicate that the maximum load was $192.7 \mathrm{kN}$, which was a decrease in load by approximately $57 \%$ compared with that of the conventional SR-joint. The load was reduced by dispersing the stress concentrated in the adjacent part of the metal and rubber rings of the conventional shape; this implies that the maximum press load to be combined was small, rendering it easier to perform molding. The purpose of this study is obtaining an optimal design that minimizes the press load for light-weighting the pressure jig and improving the lifetime of the pressure jig. The optimal design variables were set as the curling length at the top of the joint, distance between the metal and rubber rings, and jig radius.

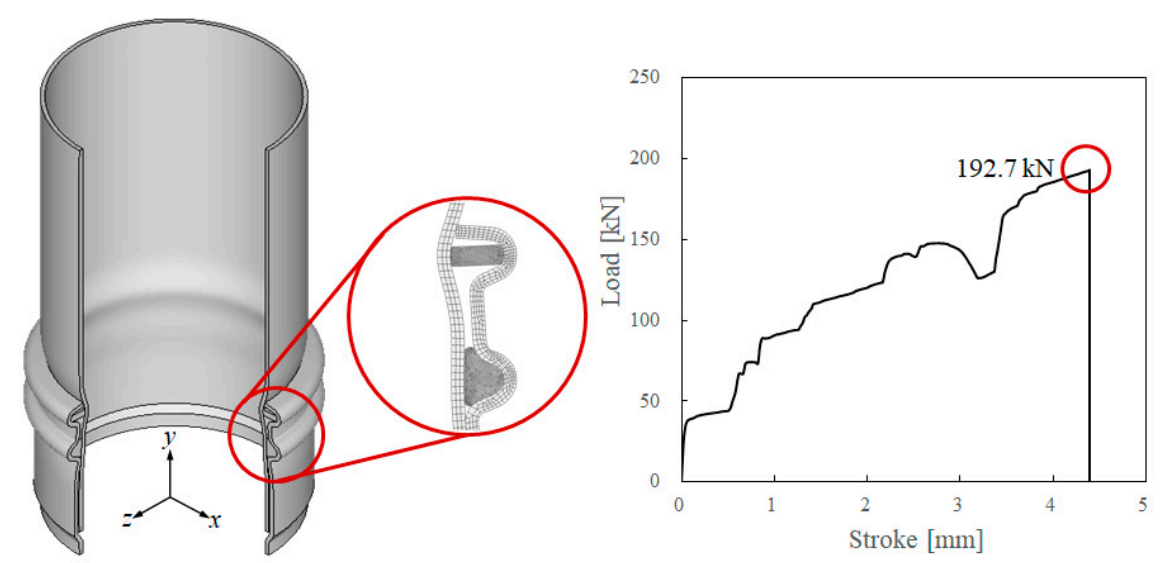

Figure 6. Numerical load-stroke curves of the proposed SR-joint (SR-II).

The defect form and the constraints of the shape based on the range of the design variables are shown in Figure 7. When the SR-joint is pressed, the metal ring should not be inclined, and the inner pipe must be deformed horizontally such that it can be compressed. It was discovered through finite element analysis that the metal ring collapsed due to compression when the metal ring was inclined and the distance was less than $6 \mathrm{~mm}$. Therefore, the distance between the metal and rubber rings was set in the range of 6-15 mm in consideration of the metal ring defects and pressure jig size. The jig radius was initially set to $4.7 \mathrm{~mm}$ based on field experience. However, the SR-joint may be dented if the jig radius is less than $4.7 \mathrm{~mm}$. In addition, the inner pipe and SR-joint may not be in contact with each other, resulting in a short shot if the jig radius exceeds $5 \mathrm{~mm}$. Therefore, the jig radius was set to $4.7-5.0 \mathrm{~mm}$. When the curling length exceeded $5.7 \mathrm{~mm}$, the metal ring was prevented from compressing the inner pipe because it was in contact with the pipe before the metal ring. If the length is less than $5.3 \mathrm{~mm}$, then the curling length will be short, rendering it impossible to prevent external impurities from flowing into the metal ring. Therefore, the curling length range was specified as $5.3-5.7 \mathrm{~mm}$. The optimum design constraint was a metal ring inclination of less than $10^{\circ}$ to withstand vibration or deflection after molding, and the upper part of the SR-joint (the curling length part) and the lower 
part of the rubber ring must be in contact with the inner pipe. This is to prevent foreign substances flowing from the upper part after connecting the SR-joint and to prevent water flowing from the lower part of the rubber ring, thereby causing corrosion.

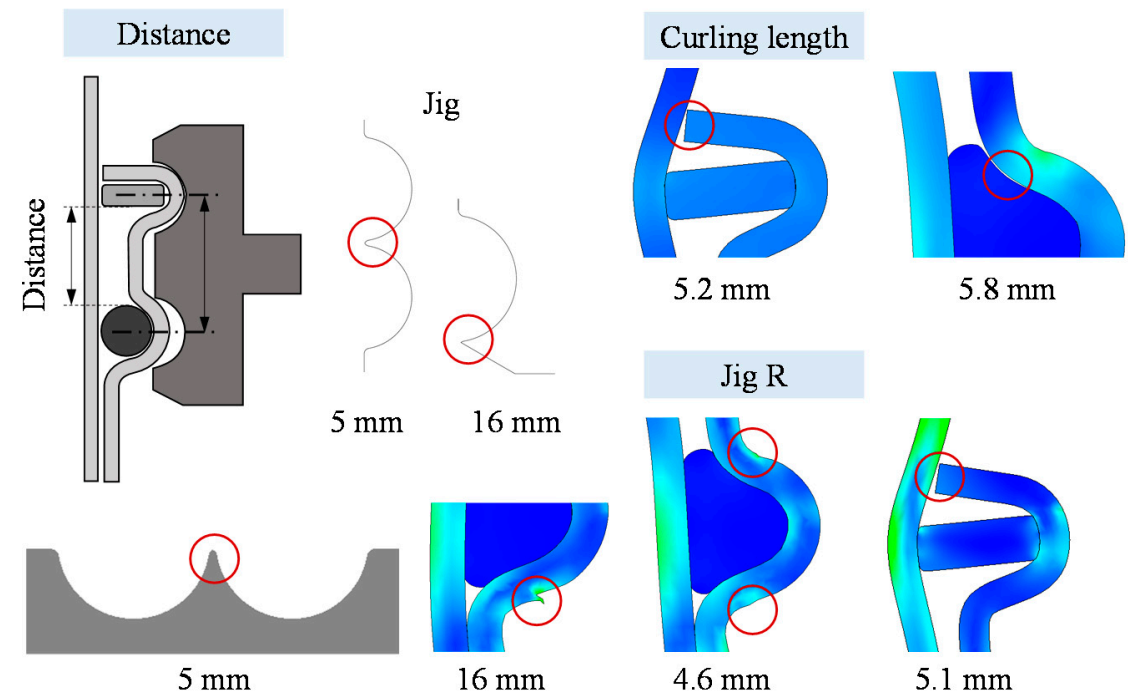

Figure 7. Shape constraints of design variables to prevent defects.

The load applied to the pressing jig was measured through finite element analysis. As for the load, the maximum load in the pressure jig was measured after finite element analysis; the load showed significant results for the design variables. Therefore, the objective function served to improve the lifetime of the pressure jig and minimize the load for light-weighting in this optimal design. The design variables, constraints, and objective functions for the optimal design are shown in Table 2, and the descriptions of the SR-joint (SR-II) analysis modeling and design variables are shown in Figure 8.

Table 2. Optimization problem set up.

\begin{tabular}{|c|c|}
\hline Design variables & $\begin{array}{c}5.3 \mathrm{~mm} \leq \text { Curling length }\left(x_{1}\right) \leq 5.7 \mathrm{~mm} \\
6.0 \mathrm{~mm} \leq \text { Distance }\left(x_{2}\right) \leq 15.0 \mathrm{~mm} \\
4.7 \mathrm{~mm} \leq \operatorname{Jig~R}\left(x_{3}\right) \leq 5.0 \mathrm{~mm}\end{array}$ \\
\hline Objective functions & $\begin{array}{c}\text { Minimize load } \\
F(x)=A x_{1}{ }^{2}+B x_{2}{ }^{2}+C x_{3}{ }^{2}+D x_{1} x_{2}+E x_{1} x_{3}+F x_{2} x_{3}+G x_{1}+H x_{2}+I x_{3}+J\end{array}$ \\
\hline Constraints & $\begin{array}{c}\text { Metal ring angle } \leq 10^{\circ} \\
\text { Contact of SR-joint and pipe }\end{array}$ \\
\hline
\end{tabular}

The full parameter case comprised 200 cases $(5 \times 10 \times 4)$ : 5 curling lengths, 10 distances, and 4 jig radii depending on the design variables. Accordingly, a finite element analysis was performed by randomly selecting 100 datasets using the "random" function. The analysis results show that the upper part of the curling did not establish a contact with pipe when the curling length was $5.3 \mathrm{~mm}$, and the jig radius was $5.0 \mathrm{~mm}$. Since this was an unsuitable shape for the product, 61 datasets were obtained excluding the defect data. The optimal design was determined using the analysis result, and the correlation discovered is as shown in Figure 9. A higher positive correlation was observed as it went from red to white from 0 in purple, and a higher absolute value indicated a higher negative correlation in the case of negative values. It was discovered through the correlation analysis that the load exhibited a positive correlation with distance and that the curling length and jig radius imposed some effects. The design variable sensitivity analysis was conducted by performing $2^{3}=8$ finite element analyses, as shown in Table 3, through factorial design considering only the maximum and minimum of each variable. The result data and the sensitivity analysis results are shown in Figure 10. The design parameter is generally set 
to 4 to understand the effect of the design parameter. A sensitivity analysis for the new objective function was performed, including the interaction of each design variable. There is study on how the optimal order of the regression equation is automatically determined in the order of minimizing the root mean square error and fitting variance when determining the regression equation [21]. Considering that the correlation of each parameter is given in the form of a general polynomial function in this study, it was expressed as a third-order polynomial. Although the regression equation is expressed in the form of a cubic equation, it shows an almost linear relationship. In the case of Jig $R$, there is vibration, but it tends to decrease slightly overall. The sensitivity analysis result shows that distance was the most sensitive to the objective function (with $21.11 \%$ ), followed by the curling length, jig radius, interaction between curling length and jig radius, interaction between curling length and distance, and interaction between distance and jig radius, with $1.85 \%, 1.34 \%, 1.31 \%, 0.35 \%$, and $0.05 \%$, respectively.

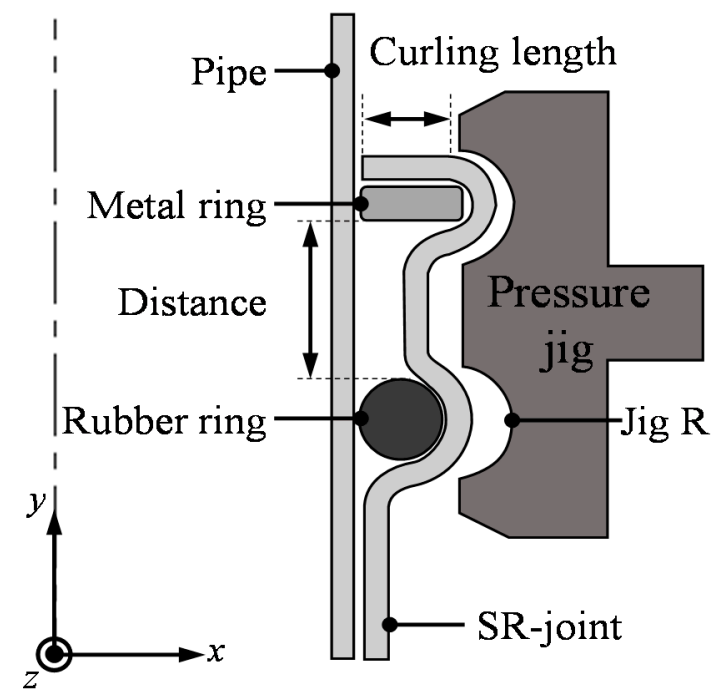

Figure 8. Design variables of the proposed SR-joint (SR-II).

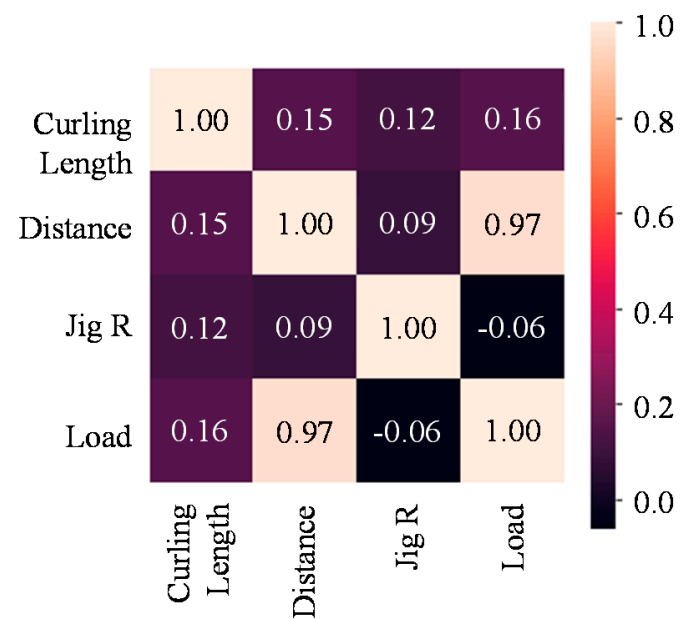

Figure 9. Comparison of correlation between design variables. 
Table 3. Dataset for sensitivity analysis.

\begin{tabular}{cccc}
\hline Curling Length $[\mathbf{m m}]$ & Distance $[\mathbf{m m}]$ & Jig R $[\mathbf{m m}]$ & Load $[\mathbf{k N}]$ \\
\hline 5.7 & 6 & 5 & 159.76 \\
5.7 & 15 & 4.7 & 196.35 \\
5.3 & 15 & 5 & 190.23 \\
5.7 & 15 & 5 & 197.91 \\
5.3 & 6 & 5 & 156.66 \\
5.3 & 6 & 4.7 & 159.40 \\
5.7 & 6 & 4.7 & 161.44 \\
5.3 & 15 & 4.7 & 198.50 \\
\hline
\end{tabular}
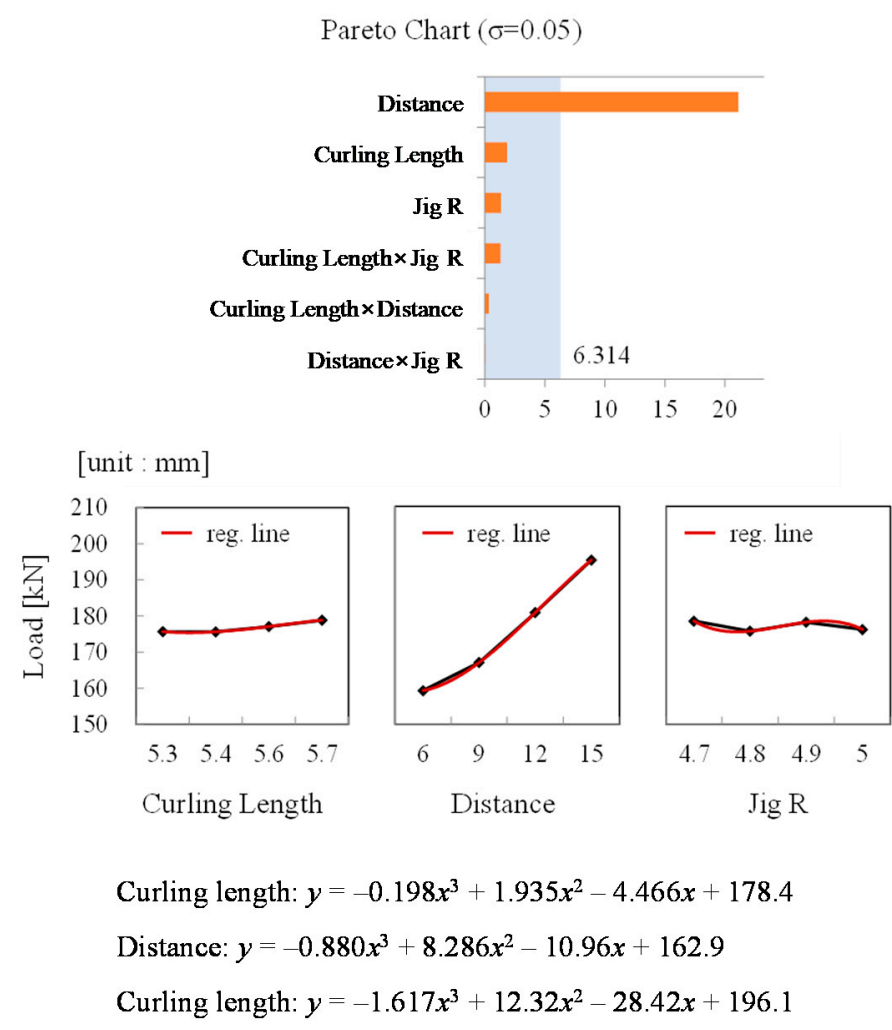

Figure 10. Result of sensitivity analysis.

\subsubsection{Optimal Design through SVM}

It was discovered through sensitivity analysis that each variable affected the objective function. Accordingly, the relationship between the objective function and design variables was predicted through machine learning. An SVM was applied during machine learning to optimize the shape for load minimization. SVM is actively used in pattern recognition and data analysis; moreover, it can be used for classification and prediction simultaneously. However, the SVM requires a significant amount of time to build the model, and it explains the results poorly. However, it has a low overfitting risk and high prediction accuracy while being easy to use [22]. Assuming that the decision boundary to be discovered in the SVM is $w^{T} x+b=0$ in Figure 11, the vector $w$ is a normal vector perpendicular to the decision boundary, and the classification and prediction methods of the SVM involve maximizing the margin surrounding the hyperplane of the decision region accordingly. The SVM is often used for classification; however, it is also actively used in regression analysis. The regression SVM was constructed based on a value obtained by converting the array in which the object was stored, and the optimal hyperplane value that minimized the margin was obtained by changing the gamma value of the kernel function built in the SVM library. 


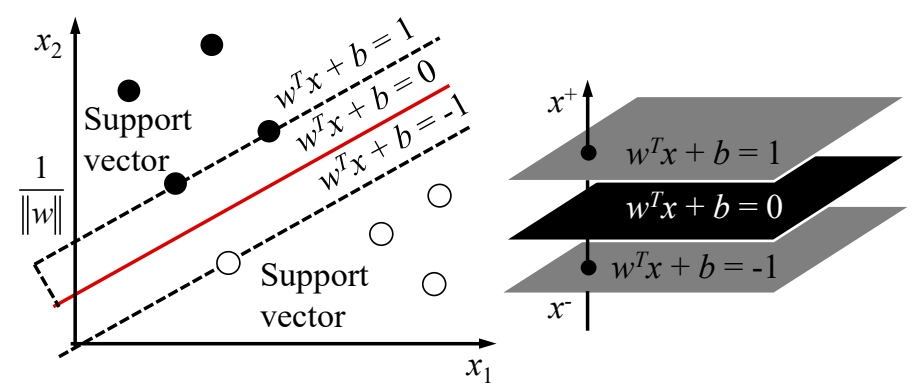

Figure 11. Concept of support vector machine.

Python 3.7 and Scikit-learn, which is a machine learning library, were used in this study. The regression method and prediction accuracy varied depending on the kernel in SVM regression. The kernels used in the SVM included linear, polynomial, and radial basis functions. The prediction accuracy analysis of each kernel based on 50 randomly selected data indicated that prediction accuracies of the radial basis function, linear, polynomial kernels were $0.93,0.91$, and 0.54 , respectively; subsequently, the radial basis kernel that yielded the highest value was used for this optimal design. The parameters used in the kernel did not exhibit a significant difference in accuracy; hence, 0.1 for gamma and 100 for $\mathrm{C}$ were applied. As for the training dataset, 61 datasets excluding the defect data were partitioned into ratios of $25 \%, 50 \%, 75 \%$, and $100 \%$ among the 100 data analyzed earlier. Meanwhile, for the test dataset, a dataset for each design variable was created, as shown in Table 4.

Table 4. Test dataset.

\begin{tabular}{cccc}
\hline Data Set & Curling Length & Distance & Jig R \\
\hline \multirow{3}{*}{ Curling length } & 5.4 & & \\
& 5.5 & 11 & 4.9 \\
& 5.6 & & \\
& 5.7 & 7 & 4.9 \\
Distance & & 9 & \\
& 5.6 & 11 & 4.7 \\
& & 13 & 4.8 \\
\hline \multirow{2}{*}{ Jig R } & & 15 & 4.9 \\
\hline
\end{tabular}

\subsubsection{Optimal Design through Statistical Regression Analysis}

The response surface method, which is a design of experiment method, was used to compare the accuracy of regression analysis between the conventional optimal design method and the method using the SVM. The response surface method was devised by Box and Wilson in 1951 and is still being used currently [23]. The response surface function can be expressed in the form of a quadratic polynomial as shown in Equation (3), where $\beta$ denotes the coefficient, and $x$ denotes the value of each design variable. Optimization was performed with an objective function that minimized the load, and the resulting equation is shown in Equation (4). The load was estimated to be $153.1 \mathrm{kN}$ when the curling length was $5.7 \mathrm{~mm}$, the distance was $6 \mathrm{~mm}$, and the jig R was $5.0 \mathrm{~mm}$.

$$
\begin{gathered}
Z=\beta_{0}+\sum_{i=1}^{n} \beta_{i} x_{i}+\sum_{i=1}^{n} \beta_{i i} x_{i}^{2}+\sum_{i<j}^{n} \beta_{i j} x_{i} x_{j} \\
Y_{\text {predict }}=(-0.2236) x_{1}^{2}+(-0.0009) x_{2}^{2}+(0.8675) x_{3}^{2}+(-0.0059) x_{1} x_{2} \\
+(0.2731) x_{1} x_{3}+(0.0045) x_{2} x_{3}+(1.2408) x_{1}+(0.0532) x_{2}+(-9.9395) x_{3}+21.0150
\end{gathered}
$$




\subsubsection{Results}

The degree of influence of the data normalization was analyzed when the optimal design was implemented to minimize the objective function load. The actual test value (Max Load), maximum normalization using the maximum value (Load-max, Load $\max$ ), maximum-minimum normalization (Load-minmax, Load $_{\text {minmax }}$ ), and the z-score method using standard deviation and average value (Load-zscore, Load $_{z s c o r e}$ ) were applied to the maximum load applied to the pressure jig. Each normalization method is expressed in Equation (5), and the predicted results for six data items through the SVM and statistical regression are shown in Figure 12, where, $y_{r}$ denotes the test value, $y_{r-\text { max }}$ denotes the maximum test value, $y_{r-\min }$ denotes the minimum test value, $\overline{y_{r}}$ denotes the average test value, and $\sigma\left(y_{r}\right)$ denotes the standard deviation of the test value.

$$
\text { Load }_{\max }=\frac{y_{r}}{y_{r-\max }}, \text { Load }_{\operatorname{minmax}}=\frac{y_{r}-y_{r-\min }}{y_{r-\max }-y_{r-\min }}, \text { Load }_{z s c o r e}=\frac{y_{r}-\bar{y}_{r}}{\sigma\left(y_{r}\right)}
$$

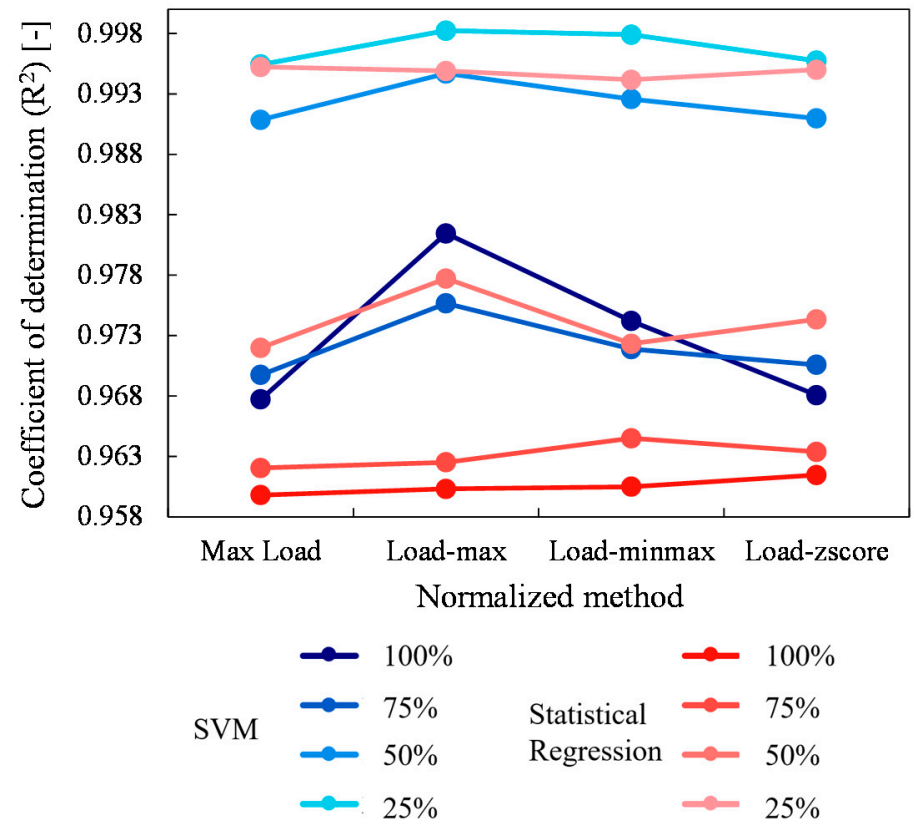

Figure 12. Comparison of prediction accuracy for support vector machine (SVM) and statistical regression data based on data normalization $\left(R^{2}\right)$.

The SVM and statistical regression analysis results are shown in blue and red, respectively. The smaller data with respect to the data ratio are shown in a lighter color, and the coefficient of determination $\left(\mathrm{R}^{2}\right)$ was used to compare the prediction accuracy. $\mathrm{R}^{2}$ was used as a measure of goodness-of-fit in the regression and calculated as shown in Equation (6), where $y_{p}$ denotes the predicted value. It was discovered in both the statistical regression and SVM that the larger data resulted a smaller $R^{2}$, and that $R^{2}$ was the highest when the maximum normalization was applied. The statistical regression analysis had a smaller $\mathrm{R}^{2}$ than the SVM regardless of the amount of data, and the maximum difference was 0.0212 when the data ratio was $100 \%$ and the maximum normalization was applied. The $\mathrm{R}^{2}$ based on the data ratio when the maximum normalization was applied is shown in Table 5 . When the data ratio was $100 \%$, the difference in $\mathrm{R}^{2}$ was the greatest at 0.0212 . The mean based on the data ratio was 0.9739 and 0.9875 for the statistical regression and SVM, respectively, and the difference was 0.0136 , indicating that the SVM was more suitable. Although the $\mathrm{R}^{2}$ was lower when the number of datasets was larger, the range was 0.0346 and 0.0225 for the statistical regression and SVM, respectively. This indicates that the relationship between the dependent and independent variables was not two-dimensional as the data ratio increased, and that the statistical regression predicted a quadratic polynomial regression equation as 
well as demonstrated the relationship between the dependent and independent variables considering only two dimensions. However, because SVM approaches involve not only two dimensions but also higher dimensions for learning the most appropriate prediction weight, they are more accurate than statistical regression analysis regardless of the amount of data. The results of training and prediction using 61 datasets are shown in Figure 13. The $\mathrm{R}^{2}$ of the SVM statistical regression is shown in blue and red, respectively; it was discovered that the SVM predicted more accurately than the statistical regression.

$$
\mathbf{R}^{2}=1-\frac{\sum\left(y_{r}-y_{p}\right)^{2}}{\sum\left(y_{r}-\bar{y}_{r}\right)^{2}}
$$

Table 5. Prediction accuracy of Load-max for SVM and statistical regression data with the number of data.

\begin{tabular}{ccc}
\hline & Coefficient of Determination \\
\hline Data Set [\%] & Statistical Regression & SVM \\
\hline 100 & 0.9603 & 0.9815 \\
75 & 0.9625 & 0.9757 \\
50 & 0.9777 & 0.9947 \\
25 & 0.9949 & 0.9982 \\
\hline
\end{tabular}

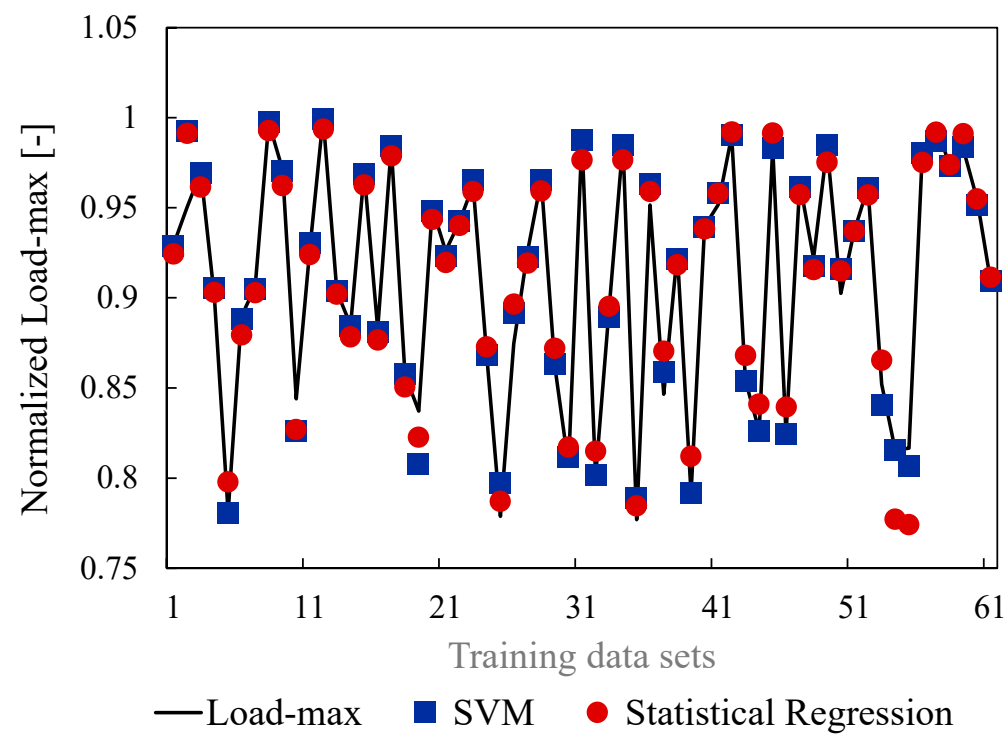

Figure 13. Normalized Load-max vs. training datasets for different regression method.

The test was conducted using the test dataset for each design variable listed in Table 4 based on the training and prediction results for each data ratio through regression analysis and the SVM. The data were compared by applying test values, maximum normalization, minimum-maximum normalization, and z-score normalization, as shown in Figure 14, based on the amount of data. Similar to the training result, the results of the SVM and statistical regression are shown in blue and red, respectively, and they were depicted in a lighter color as the data size decreased. The verification results were compared with the mean square error (MSE), as calculated using Equation (7). The MSE is the mean square error between the predicted and test values; it is generally used as a measure of predictive accuracy. The verification result shows that the normalization technique yielded the lowest MSE when the maximum normalization was applied, and the value predicted by the SVM was lower than that by the statistical regression. This shows that the SVM had higher prediction accuracy than the statistical regression and that it did not significantly affect the 
amount of data. The case where maximum normalization was applied by subdividing each design variable (see Figure 15) is depicted in blue for SVM and in red for the statistical regression. The SVM was more accurate than the statistical regression, similar with the training results. The optimal design was implemented within the limiting conditions of the design variables, and the minimum load was indicated when the curling length was $5.7 \mathrm{~mm}$, the distance was $6 \mathrm{~mm}$, and the jig radius was $5 \mathrm{~mm}$.

$$
M S E=\frac{1}{n} \sum_{i=1}^{n}\left(y_{r}-y_{p}\right)^{2}
$$

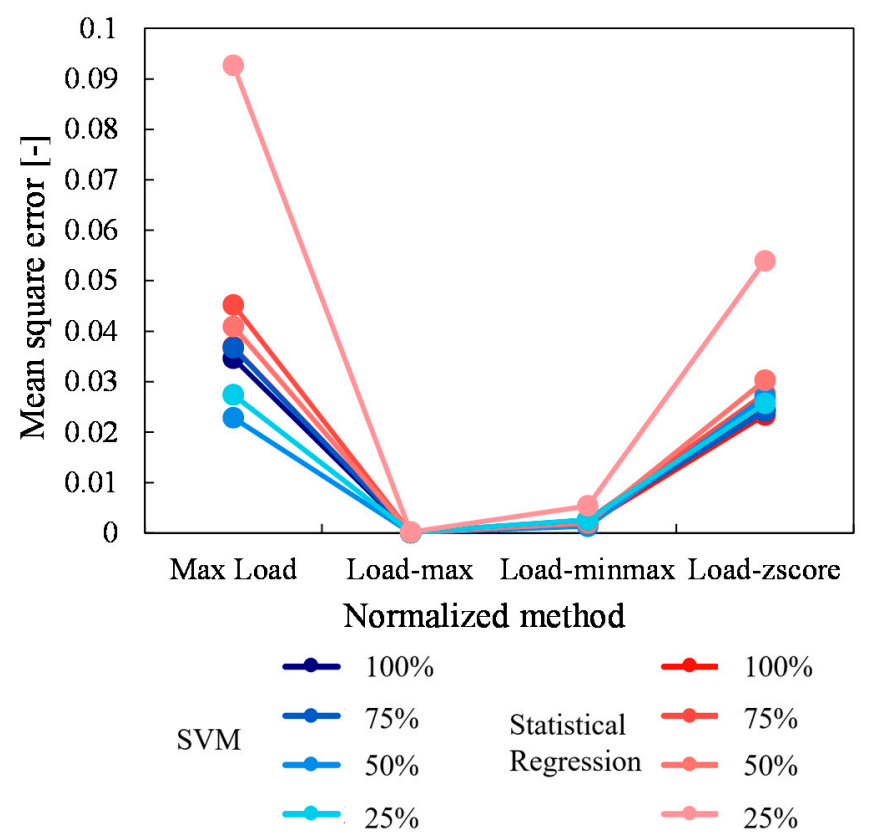

Figure 14. Comparison of mean square error for test data with SVM and statistical regression data.

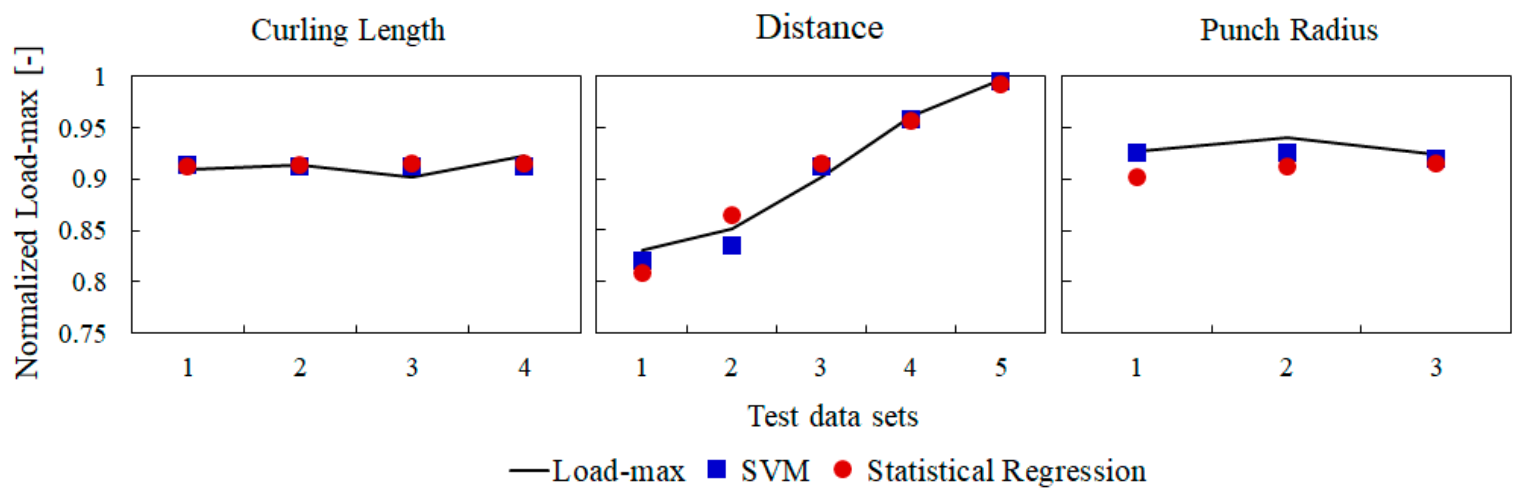

Figure 15. Normalized Load-max vs. test datasets for different regression methods.

As a result of this optimal design, molding analysis was performed when the curling length was $5.7 \mathrm{~mm}$, the distance was $6 \mathrm{~mm}$, and the jig radius was $5 \mathrm{~mm}$, and the load was discovered to be $162.2 \mathrm{kN}$. It was discovered that the molding analysis result and the predicted value were extremely similar. The load was reduced to $47.59 \%$ when comparing this value with that of the conventional SR-joint. It is believed that the water tightness and pressure jig shape, which were disadvantageous to SR-I, can be improved based on this result. In the finite element analysis result, the contact area with the mold was measured to 
be $165.74 \mathrm{~cm}^{2}$. The pressure was $9.79 \mathrm{MPa}$ when this was substituted into Equation (2), i.e., a decrease by $44.40 \%$ compared with the conventional SR-joint pressure of $22.05 \mathrm{MPa}$.

\subsection{Verification}

An actual crimping joint was designed and manufactured based on the optimal design. The FPD-8010E model of the Fujifilm pressure distribution mapping system was used to measure the stress applied to the pipe when the pipe was compressed for the accuracy verification of the molding analysis. The test verification process is shown in Figure 16a. A film was attached to the outer pipe, and compression molding was performed using a pressure jig. The color of the pressure film turned into red depending on the measurement pressure after compression molding was performed. The pressure at each point was measured using DIC equipment. The maximum pressure on the pressure film was 382 $\mathrm{MPa}$ based on the comparison result, as shown in Figure 16b. Furthermore, it was $360 \mathrm{MPa}$ based on finite element analysis, indicating the similarity between the pressure film and finite element analysis results, thereby proving the reliability of this study.

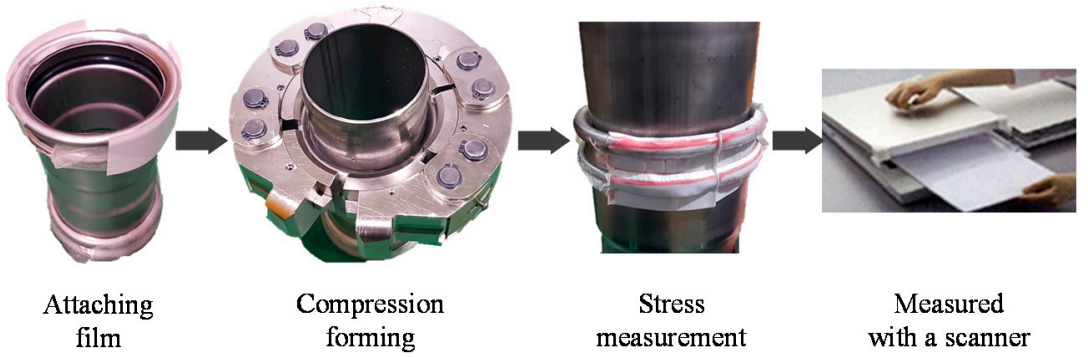

(a)

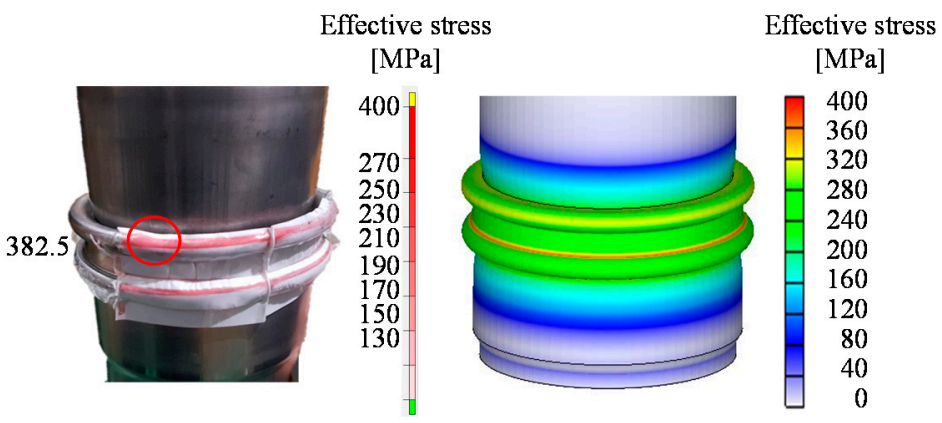

(b)

Figure 16. (a) Verification with pressure film; (b) comparison of effective stress between experiment and FE analysis.

\section{Conclusions}

In this study, a new ring-separated crimping joint was designed by separating a metal ring and a rubber ring from the conventional joint shape to minimize the corrosion of the joint crimping pipe and improve the water tightness. An optimal design was implemented using finite element analysis and an SVM to minimize the press load.

(1) To improve the water tightness and minimize the load during compression, the shape of the pressing tool and process were modeled. Finite element analysis results indicated that molding can be performed within $30 \mathrm{MPa}$, which was the maximum hydraulic pressure applied during compression molding in the field.

(2) The curling length at the top of the joint, distance between the metal ring and rubber rings, and jig radius were selected as the design variables to design the jig shape; meanwhile, the minimum load of the ring-separated crimping joint and the optimal design variables were determined using the SVM. By comparing the prediction results 
using the statistical regression method based on the response surface method and the SVM load, it was discovered that the two methods yielded similar results when the number of datasets generated through the analysis was small. However, the SVM prediction indicated higher prediction accuracy as the number increased.

(3) The curling length, distance between metal and rubber rings, and jig radius were derived to be $5.6,15$, and $4.7 \mathrm{~mm}$, respectively, for the shape obtained from the optimal design. Subsequently, a crimping joint with dual ring points with an optimized shape was obtained to verify the accuracy of the analysis, and a pressure film was attached to measure the pressure distribution and then compare it with the optimal design value. A comparison of the pressure distributions outside the joint showed that maximum pressure distributions of 382 and $360 \mathrm{MPa}$ were achieved in the actual product and finite element analysis, respectively, thereby proving the reliability of this study.

Author Contributions: Conceptualization, S.H.; methodology, M.K. and S.Y. and S.H.; software, M.K. and S.Y.; validation, M.K.; investigation, M.K. and S.Y.; writing-original draft preparation, M.K. and S.Y.; writing—review and editing, S.H.; visualization, M.K. and S.Y.; supervision, S.H.; project administration, S.H. All authors have read and agreed to the published version of the manuscript.

Funding: This research received no external funding.

Institutional Review Board Statement: Not applicable.

Informed Consent Statement: Not applicable.

Data Availability Statement: The data presented in this study are available in article.

Conflicts of Interest: The authors declare no conflict of interest.

\section{References}

1. Yoon, K.; Kim, S.; Kang, J. Development of new dual ring press joint for the water pipe. In Proceedings of the Korean Society for Marine Environment and Energy, KOSMEE Autumn Annual Conference, Seogwipo, Korea, 11-12 October 2017.

2. Onyegiri, I.; Kashtalyan, M. Finite element analysis of a sandwich pipe joint. Ocean Eng. 2017, 146, 363-374. [CrossRef]

3. Li, J.; Zhou, C.Y.; Zhu, J.G. Limit loads for $180^{\circ}$ pipe bends under in-plane bending moment considering geometric nonlinearity. Int. J. Press. Vessel. Pip. 2020, 183, 1-13. [CrossRef]

4. Lee, H.S.; Lee, Y.S.; Baek, J.H.; Chun, B.S.; Kim, S.Y.; Lee, J. Contact analysis of a wedge-type pipe joint. In Proceedings of the Korean Society of Mechanical Engineers, KSME Autumn Conference, Pyeongchang, Korea, 31 October-2 November 2007.

5. Lee, H.J.; Ryu, H.Y.; Kim, H.J.; Jo, H.H.; Bae, S.B. Manufacturing technology of press die for compression-type pipe joint. In Proceedings of the Korean Society for Precision Engineering, KSPE Spring Conference, Jeju, Korea, 26-28 May 2010.

6. Lee, J.H.; Bae, S.B.; Youn, K.T.; Heo, H.Y.; Kim, S.H.; Park, C.D. Wear life prediction of CrN coating layer on the press tool for stamping the ultra-high strength steel sheet. Trans. Mater. Process. 2017, 26, 137-143. [CrossRef]

7. Hosseinpour, V.; Kazemeini, M.; Rashidi, A. Developing a metamodel based upon thew DOE approach for investigating the overall performance of microchannel heat sinks utilizing a variety of internal fins. Int. J. Heat Mass Tran. 2020, 149, 1-20. [CrossRef]

8. Son, D.G.; Chung, W.J. Prediction model of surface deflection of rectangular drawing products using finite element analysis and machine learning. Trans. Korean Soc. Mech. Eng. A 2019, 43, 41-50. [CrossRef]

9. Kwak, M.J.; Park, J.W.; Park, K.T.; Kang, B.S. A development of optimal design model for initial blank shape using artificial neural network in rectangular case forming with large aspect ratio. Trans. Mater. Process. 2020, 29, 272-281. [CrossRef]

10. Kwak, M.J.; Park, J.W.; Park, K.T.; Kang, B.S. A development of longitudinal and transverse springback prediction model using artificial neural network in multipoint dieless forming of advanced high strength steel. Trans. Mater. Process. 2020, $29,76-88$. [CrossRef]

11. Woo, M.A.; Lee, S.M.; Lee, K.H.; Song, W.J.; Kim, J. Application of an artificial neural network model to obtain constitutive equation parameters of materials in high-speed forming process. Trans. Mater. Process. 2018, 27, 331-338. [CrossRef]

12. Park, J.W.; Kang, B.S. Comparison between regression and artificial neural network for prediction model of flexibly reconfigurable roll forming process. Int. J. Adv. Manuf. Tech. 2019, 101, 3081-3091. [CrossRef]

13. Wang, Z.H.; Gong, D.Y.; Li, X.; Li, G.T.; Zhang, D.H. Prediction of bending force in the hot strip rolling process using artificial neural network and genetic algorithm (ANN-GA). Int. J. Adv. Manuf. Tech. 2017, 93, 3325-3338. [CrossRef]

14. Park, E.T.; Lee, Y.H.; Kim, J.; Kang, B.S.; Song, W.J. Numerical prediction of temperature-dependent flow stress on fiber metal laminate using artificial neural network. Trans. Mater. Process. 2018, 27, 227-235. [CrossRef]

15. Olazagoitia, J.L.; Perez, J.A.; Badea, F. Identification of tire model parameters with artificial neural networks. Appl. Sci. 2020, 10, 9110. [CrossRef] 
16. Mei, H.; Lang, L.; Li, X.; Mirza, H.A.; Yang, X. Prediction of tensile strength and deformation of diffusion bonding joint for Inconel 718 using deep neural network. Metals 2020, 10, 1266. [CrossRef]

17. Hijazi, A.; Al-Dahidi, S.; Altarazi, S. Residual strength prediction of aluminum panels with multiple site damage using artificial neural networks. Materials 2020, 13, 5216. [CrossRef] [PubMed]

18. Jiang, J.; Xu, J.S.; Zhang, Z.S.; Chen, X. Rate-dependent compressive behavior of EPDM insulation: Experimental and constitutive analysis. Mech. Mater. 2016, 96, 30-38. [CrossRef]

19. KS B 0801. Test pieces for tensile test for metallic materials. In Korean Industrial Standards; Korean Standards Association: Seoul, Korea, 2017.

20. Fernandes, J.V.; Rodrigues, D.M.; Menezes, L.F.; Vieira, M.F. A modified swift law for pre-strained materials. Int. J. Plast. 1998, 14, 537-550. [CrossRef]

21. Kamiński, M.; Świta, P. Structural stability and reliability of the underground steel tanks with the stochastic finite element method. Arch. Civ. Mech. Eng. 2015, 15, 593-602. [CrossRef]

22. Zgheib, W.A.; Barbar, A.M. A study using support vector machines to classify the sentiments of tweets. Int. J. Comput. Appl. 2017, 170, 8-12. [CrossRef]

23. Box, G.E.P.; Wilson, K.B. On the experimental attainment of optimum conditions. J. R. Stat. Soc. 1951, 13, 1-45. [CrossRef] 